\title{
A Molecular Approach to Breast Imaging
}

\author{
Amy M. Fowler \\ Department of Radiology, University of Wisconsin-Madison, Madison, Wisconsin
}

\begin{abstract}
Molecular imaging is a multimodality discipline for noninvasively visualizing biologic processes at the subcellular level. Clinical applications of radionuclide-based molecular imaging for breast cancer continue to evolve. Whole-body imaging, with scintimammography and PET, and newer dedicated breast imaging systems are reviewed. The potential clinical indications and the challenges of implementing these emerging technologies are presented.
\end{abstract}

Key Words: molecular imaging; oncology; breast; PET; PET/ CT; radiopharmaceuticals; breast cancer; breast-specific $\gamma$ imaging; positron-emission mammography; positron-emission tomography

J Nucl Med 2014; 55:177-180

DOI: 10.2967/jnumed.113.126102

Molecular imaging allows noninvasive visualization of the underlying pathobiology of cancer. It holds promise for personalized medicine by detecting the presence of specific biologic targets used to tailor therapy and predict outcome. Additionally, molecular imaging can be beneficial for new drug development using an imaging parameter to serve as a surrogate endpoint for survival in clinical trials, saving time and cost. This article concentrates on the clinical applications of molecular imaging for breast cancer, focusing on radionuclide techniques.

\section{TYPES OF MOLECULAR IMAGING USED FOR BREAST CANCER}

The potential for molecular imaging of breast cancer was discovered serendipitously in the 1970s when single-photonemitting radiopharmaceuticals administered for bone and cardiac imaging also localized within breast malignancies (1). Further investigations of planar scintigraphy and SPECT of the breast, termed scintimammography, have focused primarily on ${ }^{99 \mathrm{~m}} \mathrm{Tc}$-methoxyisobutylisonitrile ( ${ }^{99 \mathrm{~m}} \mathrm{Tc}$-sestamibi).

${ }^{99 m}$ Tc-sestamibi accumulation in cancer reflects increased vascularity and concentration of mitochondria in

Received Oct. 1, 2013; revision accepted Dec. 19, 2013.

For correspondence or reprints contact: Amy M. Fowler, Department of Radiology, University of Wisconsin-Madison, 600 Highland Ave., Madison, WI 53792-3252.

E-mail: afowler@uwhealth.org

Published online Jan. 16, 2014.

COPYRIGHT (C) 2014 by the Society of Nuclear Medicine and Molecular Imaging, Inc. malignant cells. A recent meta-analysis of the accuracy of ${ }^{99 m}$ Tc-sestamibi scintimammography as an adjunct to diagnostic mammography for detection of breast cancer demonstrated a sensitivity of $83 \%$ and specificity of $85 \%$ (2). However, sensitivity was less for nonpalpable (59\%) versus palpable lesions (87\%) despite comparable specificity, with no significant difference between planar and SPECT methods. Decreased sensitivity for nonpalpable, presumably smaller, lesions is in part due to the limited spatial resolution of conventional $\gamma$ cameras.

In addition to ${ }^{99 \mathrm{~m}} \mathrm{Tc}$-sestamibi, the positron-emitting radiopharmaceutical ${ }^{18}$ F-FDG accumulates in many types of cancer including breast. Meta-analyses of the accuracy of whole-body ${ }^{18}$ F-FDG PET used after standard diagnostic workup for patients with suspected breast lesions demonstrated sensitivities of $83 \%-89 \%$ and specificities of $74 \%-$ $80 \%(3,4)$. For patients with newly diagnosed biopsyproven breast cancer, the sensitivity of combined PET/CT was $87 \%(5,6)$. However, sensitivity is less than $50 \%$ for small $(\leq 1 \mathrm{~cm})$, low-grade invasive cancers and ductal carcinoma in situ (DCIS). Thus, current recommendations do not support the routine use of ${ }^{18} \mathrm{~F}$-FDG PET for primary breast cancer diagnosis because of its false-negative risk and have encouraged the development of dedicated breast PET scanners with improved spatial resolution for imaging small, clinically relevant, cancers (4).

\section{Single-Photon $\gamma$ Imaging Systems for Dedicated Breast Imaging}

For this review, dedicated breast imaging systems designed for single-photon-emitting radiopharmaceuticals will be collectively referred to as breast-specific $\gamma$ imaging (BSGI). These systems use standard mammographic positioning (craniocaudal and mediolateral oblique) with the breast in direct contact with the $\gamma$ camera. They differ mainly by the type (scintillation crystal vs. semiconductor) and configuration (single vs. dual) of the detector (7). Commercially available BSGI systems include the Dilon 6800 (Dilon Diagnostics), LumaGEM (Gamma Medica), and Discovery NM750b (GE Healthcare).

${ }^{99 m}$ Tc-sestamibi is the only single-photon-emitting radiopharmaceutical that has approval from the Food and Drug Administration (FDA) for breast imaging. It is approved as a diagnostic tool after mammography and is not indicated for screening or as an alternative to biopsy. Typical protocols specify an injection of 740-1,100 MBq (20-30 mCi) 
into the arm contralateral to the suspected abnormality. Imaging begins 5-10 min after injection, with 10 min per view.

A meta-analysis of the accuracy of BSGI as an adjunct to mammography for breast cancer detection in both the diagnostic and the high-risk screening settings demonstrated a sensitivity of $95 \%$ and specificity of $80 \%(8)$. Sensitivity decreased for subcentimeter cancers $(84 \%)$ and DCIS $(88 \%)$ but was improved compared with prior reports of conventional scintimammography. Common causes of false-positives included fibrocystic changes, fibroadenomas, and benign breast tissue not otherwise specified. No studies directly compared conventional scintimammography with BSGI.

\section{Annihilation Coincidence Detection Systems for Dedicated Breast Imaging}

Several dedicated breast imaging systems for positronemitting radiopharmaceuticals have been designed $(1,7)$. These systems differ by the geometry and mobility of the detectors (stationary flat, rotating flat, stationary full- or partial-ring) and by the positioning/immobilization of the breast. The earliest design uses 2 flat detectors placed on opposite sides of the breast in mild compression with the patient seated. Given that its positioning is similar to that of mammography, this design has been termed positronemission mammography (PEM). An alternative design, termed dedicated breast PET, exists in which the patient is prone and the breast hangs freely through a small ring of detectors.

The first system to become commercially available was the Flex Solo II PEM (Naviscan Inc.). It is a stationary flat detector-based PEM scanner using limited-angle tomosynthetic reconstruction with an in-plane spatial resolution of $2.4 \mathrm{~mm}$, a marked improvement over whole-body PET/CT ( $\sim-7 \mathrm{~mm}$ ) (9). Because images are obtained in the same projections as mammography with minimal breast compression, correlation of imaging findings can be performed with direct coregistration possible using the earlier integrated PEM-I design. The Flex Solo II has approval from the FDA and has been the most extensively studied with multicenter trials.

A newer, alternative design that is commercially available is MAMmography with Molecular Imaging (MAMMI) dedicated breast PET (Oncovision). It uses a complete ring of detectors for full tomographic image reconstruction with a spatial resolution of $1.6 \mathrm{~mm}(10)$. This system has certification in Europe, and the company expects to have FDA approval in 2014.

The typical protocol for dedicated breast imaging systems uses an intravenous dose of $370 \mathrm{MBq}(10 \mathrm{mCi})$ of ${ }^{18} \mathrm{~F}-\mathrm{FDG}$ for fasting patients. Image acquisition begins 60-120 min after injection and lasts approximately $20 \mathrm{~min} /$ breast (10 $\mathrm{min} /$ craniocaudal or mediolateral oblique view) for PEM and 5-15 min/breast for MAMMI PET.

No meta-analysis of the diagnostic performance of dedicated breast PET/PEM systems has been published yet. Pooled data from several studies of PEM for patients with suspected breast lesions yielded a sensitivity of $87 \%$ and specificity of $85 \%$ (1). Sensitivities for subcentimeter cancers and DCIS were $73 \%$ and $88 \%$, respectively, which exceed those previously reported for whole-body PET. Furthermore, several studies confirmed the improved sensitivity of PEM (92\%-95\%) when directly compared with whole-body PET $(56 \%-68 \%)$ or PET/CT $(87 \%)$ obtained after a single injection of ${ }^{18} \mathrm{~F}$-FDG (Fig. 1) $(5,6,11)$. Thus, the proposed advantage of dedicated breast imaging in detecting small cancers, compared with whole-body imaging systems, holds true.

\section{CLINICAL INDICATIONS}

Initial studies introduced molecular imaging as diagnostic adjuncts to conventional dedicated breast imaging using FDA-approved radiopharmaceuticals and imaging devices with codes for potential reimbursement. However, further clarification of the appropriate clinical indications is needed to support their adoption into routine practice. Proposed indications include presurgical planning, response to therapy monitoring, evaluation for recurrence, and adjunctive screening for women with dense breasts.

\section{Preoperative Evaluation of Disease Extent}

The use of imaging to guide surgical management (lumpectomy vs. mastectomy) involves careful evaluation of additional suggestive mammographic or sonographic findings with potential for additional biopsies to prove extent of disease. Because of its excellent sensitivity, dynamic contrast-enhanced breast MR imaging has been well-studied as an adjunctive imaging modality for surgical planning. A recent meta-analysis revealed that MR imaging detects additional malignancy in $20 \%$ of patients, which included 5\% in the contralateral breast (12). However, there remains lack of evidence demonstrating improved patient outcomes when MR imaging is used. Additionally, MR imaging's high sensitivity is not as equally matched by its specificity, resulting in false-positive findings that can further delay definitive treatment.

The potential improved specificity of dedicated breast molecular imaging prompted several studies comparing preoperative BSGI and PEM with MR imaging. Small, retrospective, single-site studies using BSGI report detection of additional malignancy in $9 \%-11 \%$ of patients, which included $3 \%$ with a contralateral occult cancer (13-15). One report that directly compared BSGI with MR imaging examined 66 patients and demonstrated comparable sensitivity ( $89 \%$ vs. $92 \%$, respectively) with increased specificity of BSGI (90\%) over MR imaging (39\%) (16). Another report that directly compared preoperative PEM with MR imaging was a large, prospective, multicenter study of 388 women that found greater specificity with PEM (91\% PEM vs. $86 \%$ MR imaging, $P=$ 0.03 ) and comparable breast-level sensitivity for additional unsuspected cancers found with MR imaging (60\%) or PEM (51\%) (17). Both studies support the use of molecular 


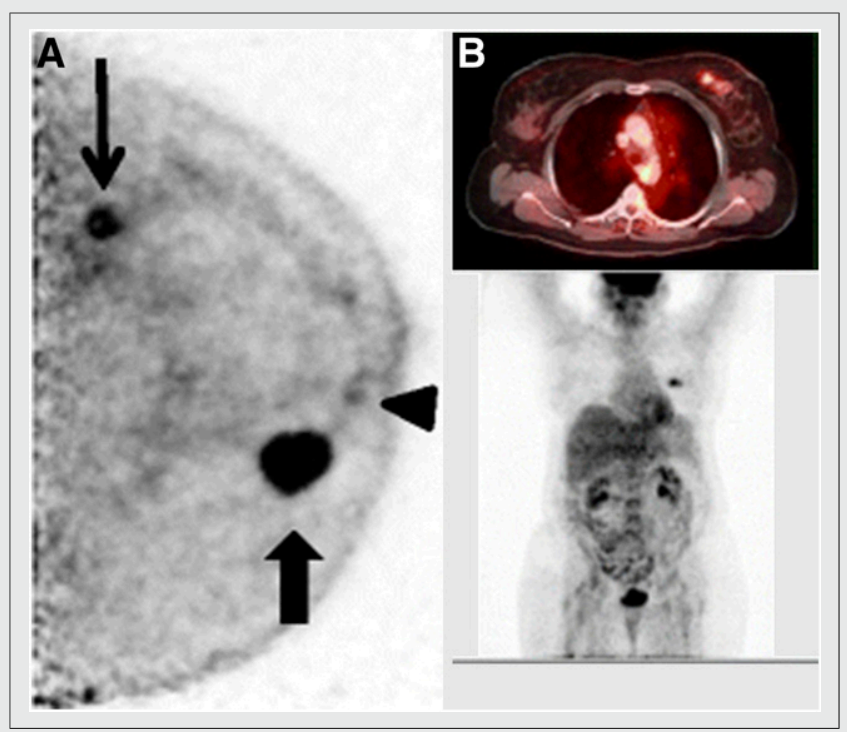

FIGURE 1. Increased sensitivity of dedicated breast molecular imaging. (A) PEM identified $2.1-\mathrm{cm}$ index cancer in anterior left breast (large arrow), $0.9-\mathrm{cm}$ mass in posterior breast (small arrow), and $0.4-\mathrm{cm}$ focus of abnormal retroareolar uptake (arrowhead). Mastectomy yielded $2.2-\mathrm{cm}$ grade III invasive ductal carcinoma, focus of DCIS in posterior specimen, and atypical ductal hyperplasia behind nipple. (B) Latter 2 lesions were not seen on whole-body PET/CT. (Reprinted with permission from (5).)

imaging as a valid alternative to MR imaging when MR imaging has limited access or is not tolerated by the patient.

\section{Response to Neoadjuvant Therapy}

Neoadjuvant systemic therapy can be used for patients with newly diagnosed breast cancer before surgery to reduce the tumor size and allow conversion from mastectomy to lumpectomy and can predict disease-free survival if complete pathologic response is achieved. Another advantage of neoadjuvant therapy is its use in clinical trial designs as a surrogate endpoint for survival, which makes testing new drugs cheaper and faster than the traditional adjuvant approach, which may take several decades to demonstrate a difference. Thus, accurate quantitative imaging is key for evaluating response to neoadjuvant therapy.

Dynamic contrast-enhanced MR imaging has been demonstrated to be a useful tool for determining patient response to neoadjuvant chemotherapy (18). It has been shown to be more accurate than mammography and physical examination, with high specificity $(91 \%)$ and moderate sensitivity $(63 \%)$ for predicting complete pathologic response (19). Potential reasons for predictive errors include fibrosis or scar formation and tumor fragmentation instead of concentric shrinkage, which can underestimate response, and antiangiogenic effects of the chemotherapeutic agent on contrast kinetics, which can overestimate response.

Measuring functional changes in tumor metabolic activity, instead of anatomic changes, as a response to therapy has also been studied. A recent meta-analysis demonstrated moderately high sensitivity (80\%) and specificity (79\%) of whole-body ${ }^{18}$ F-FDG PET or PET/CT in early differentia- tion of responders from nonresponders (20). Although there are no currently published reports of the use of PEM or dedicated breast PET for evaluating response to neoadjuvant chemotherapy, at least one clinical trial is under way (NCT01012440). Unfortunately, BSGI has shown limited predictive value in a recent study of 20 patients, which may be due to its reduced uptake in cancers expressing the multidrug resistant P-glycoprotein (21).

\section{Screening of Women with Dense Breasts}

Mammography is the only modality proven by multiple large, randomized controlled trials to have a mortality benefit. A drawback of mammography, however, is decreased sensitivity with increased breast density. Adjunctive modalities such as ultrasound, MR imaging, or BSGI have been proposed to improve the sensitivity of mammography for screening women with dense breasts.

The largest prospective study of BSGI involved 936 women with dense breasts and at least one risk factor (22). They found that the addition of BSGI increased the sensitivity from $27 \%$ to $91 \%$ and increased the cancer detection rate by 7.5 per 1,000 women screened. A recognized limitation is the increased radiation exposure from a $740-\mathrm{MBq}$ (20-mCi) dose of ${ }^{99 m}$ Tc-sestamibi (6.5-mSv effective dose equivalent for BSGI vs. $0.7 \mathrm{mSv}$ for mammography). Because of the radiation risk with the current protocol, the American College of Radiology does not recommend BSGI or PEM for breast cancer screening (23). However, a reduced dose of ${ }^{99 m} \mathrm{Tc}$-sestamibi $(2 \mathrm{mCi}$, or $74 \mathrm{MBq}$ ) would yield an effective dose comparable to that of mammography. Studies validating the diagnostic performance of BSGI at lower doses are ongoing.

\section{CHALLENGES AND FUTURE DIRECTIONS}

Several barriers exist to the widespread implementation of molecular imaging for breast cancer. Most important, current techniques confer an effective (whole-body) radiation dose 10-20 times higher than mammography (1). Further, standardized interpretive criteria must be used. Although proposed lexicons for BSGI and PEM have been published, they have not yet been incorporated into the $B I$ RADS Atlas (24-26). Additionally, there must be a biopsy method for findings seen only with molecular imaging. This has recently been addressed with the development of FDAapproved accessory biopsy units for both BSGI and PEM. Last, continued prospective clinical trials addressing the comparative effectiveness with conventional imaging are needed, particularly for evaluating response to therapy.

Molecular imaging has the power of using a spectrum of radiopharmaceuticals beyond sestamibi and ${ }^{18} \mathrm{~F}-\mathrm{FDG}$ to probe different tumor phenotypes that may be important for prognosis and predicting treatment response. For example, the following PET tracers can be used to assess general tumor characteristics: ${ }^{18} \mathrm{~F}$-fluorothymidine for proliferation, ${ }^{18} \mathrm{~F}$-fluoromisonidazole for hypoxia, ${ }^{11} \mathrm{C}$-methionine for protein synthesis, and ${ }^{11} \mathrm{C}$-choline for membrane synthesis. 
Specific imaging of tumor receptors known to be important prognostic and predictive biomarkers include ${ }^{18} \mathrm{~F}$-fluoroestradiol for estrogen receptor, ${ }^{18} \mathrm{~F}$-fluoro furanyl norprogesterone for progesterone receptor, and ${ }^{89} \mathrm{Zr}$-trastuzumab for human epidermal growth factor receptor 2 .

\section{CONCLUSION}

Molecular imaging holds promise as an important adjunct modality for breast cancer detection and characterization. As a diagnostic tool, however, it should be used only after a complete standard imaging evaluation and should not be used as an alternative to biopsy. It appears to be most useful when MR imaging is not an option. Molecular imaging holds particular promise for monitoring response to neoadjuvant therapy. Investigational radiopharmaceuticals have the added potential to reveal underlying cancer biology and thus guide selection of targeted therapy and monitor response. Critical future research necessary for further evolution of molecular imaging is radiation dose reduction and prospective clinical trials comparing its effectiveness with conventional imaging. Molecular imaging has tremendous potential for new advances in earlier detection and expanding knowledge into the pathobiologic processes used for accurate prognosis and tailored treatment, with the hope of ultimately improving patient survival.

\section{DISCLOSURE}

No potential conflict of interest relevant to this article was reported.

\section{REFERENCES}

1. Hruska CB, O'Connor MK. Nuclear imaging of the breast: translating achievements in instrumentation into clinical use. Med Phys. 2013;40:050901.

2. Xu HB, Li L, Xu Q. Tc-99m sestamibi scintimammography for the diagnosis of breast cancer: meta-analysis and meta-regression. Nucl Med Commun. 2011;32: 980-988.

3. Bruening W, Uhl S, Fontanarosa J, Reston J, Treadwell J, Schoelles K. Noninvasive Diagnostic Tests for Breast Abnormalities: Update of a 2006 Review. Comparative effectiveness review no. 47. AHRQ publication 12-EHC014-EF. Rockville, MD: Agency for Healthcare Research and Quality; 2012:27-34.

4. Fletcher JW, Djulbegovic B, Soares HP, et al. Recommendations on the use of ${ }^{18}$ F-FDG PET in oncology. $J$ Nucl Med. 2008;49:480-508.

5. Kalinyak JE, Berg WA, Schilling K, Madsen KS, Narayanan D, Tartar M. Breast cancer detection using high-resolution breast PET compared to whole-body PET or PET/CT. Eur J Nucl Med Mol Imaging. October 2, 2013 [Epub ahead of print].

6. Eo JS, Chun IK, Paeng JC, et al. Imaging sensitivity of dedicated positron emission mammography in relation to tumor size. Breast. 2012;21:66-71.
7. Surti S. Radionuclide methods and instrumentation for breast cancer detection and diagnosis. Semin Nucl Med. 2013;43:271-280.

8. Sun Y, Wei W, Yang HW, Liu JL. Clinical usefulness of breast-specific gamma imaging as an adjunct modality to mammography for diagnosis of breast cancer: a systemic review and meta-analysis. Eur J Nucl Med Mol Imaging. 2013;40: 450-463.

9. MacDonald L, Edwards J, Lewellen T, Haseley D, Rogers J, Kinahan P. Clinical imaging characteristics of the positron emission mammography camera: PEM Flex Solo II. J Nucl Med. 2009;50:1666-1675.

10. Moliner L, Gonzalez AJ, Soriano A, et al. Design and evaluation of the MAMMI dedicated breast PET. Med Phys. 2012;39:5393-5404.

11. Schilling K, Narayanan D, Kalinyak JE, et al. Positron emission mammography in breast cancer presurgical planning: comparisons with magnetic resonance imaging. Eur J Nucl Med Mol Imaging. 2011;38:23-36.

12. Plana MN, Carreira C, Muriel A, et al. Magnetic resonance imaging in the preoperative assessment of patients with primary breast cancer: systematic review of diagnostic accuracy and meta-analysis. Eur Radiol. 2012;22:26-38.

13. Brem RF, Shahan C, Rapleyea JA, et al. Detection of occult foci of breast cancer using breast-specific gamma imaging in women with one mammographic or clinically suspicious breast lesion. Acad Radiol. 2010;17:735-743.

14. Killelea BK, Gillego A, Kirstein LJ, et al. George Peters Award: how does breast-specific gamma imaging affect the management of patients with newly diagnosed breast cancer? Am J Surg. 2009;198:470-474.

15. Zhou M, Johnson N, Gruner S, et al. Clinical utility of breast-specific gamma imaging for evaluating disease extent in the newly diagnosed breast cancer patient. Am J Surg. 2009;197:159-163.

16. Kim BS. Usefulness of breast-specific gamma imaging as an adjunct modality in breast cancer patients with dense breast: a comparative study with MRI. Ann Nucl Med. 2012;26:131-137.

17. Berg WA, Madsen KS, Schilling K, et al. Breast cancer: comparative effectiveness of positron emission mammography and MR imaging in presurgical planning for the ipsilateral breast. Radiology. 2011;258:59-72.

18. Hylton NM, Blume JD, Bernreuter WK, et al. Locally advanced breast cancer: MR imaging for prediction of response to neoadjuvant chemotherapy-results from ACRIN 6657/I-SPY TRIAL. Radiology. 2012;263:663-672.

19. Yuan Y, Chen XS, Liu SY, Shen KW. Accuracy of MRI in prediction of pathologic complete remission in breast cancer after preoperative therapy: a metaanalysis. AJR. 2010;195:260-268.

20. Mghanga FP, Lan X, Bakari KH, Li C, Zhang Y. Fluorine-18 fluorodeoxyglucose positron emission tomography-computed tomography in monitoring the response of breast cancer to neoadjuvant chemotherapy: a meta-analysis. Clin Breast Cancer. 2013;13:271-279.

21. Wahner-Roedler DL, Boughey JC, Hruska CB, et al. The use of molecular breast imaging to assess response in women undergoing neoadjuvant therapy for breast cancer: a pilot study. Clin Nucl Med. 2012;37:344-350.

22. Rhodes DJ, Hruska CB, Phillips SW, Whaley DH, O'Connor MK. Dedicated dualhead gamma imaging for breast cancer screening in women with mammographically dense breasts. Radiology. 2011;258:106-118.

23. Mainiero MB, Lourenco A, Mahoney MC, et al. ACR appropriateness criteria breast cancer screening. J Am Coll Radiol. 2013;10:11-14.

24. Conners AL, Maxwell RW, Tortorelli CL, et al. Gamma camera breast imaging lexicon. AJR. 2012;199:W767-W774.

25. Narayanan D, Madsen KS, Kalinyak JE, Berg WA. Interpretation of positron emission mammography: feature analysis and rates of malignancy. AJR. 2011; 196:956-970.

26. BI-RADS Breast Imaging and Reporting Data System: Breast Imaging Atlas. 4th ed. Reston, VA: American College of Radiology; 2003. 